\title{
Correction to: Fish vs. Aliens: predatory fish regulate populations of Limnoperna fortunei mitigating impacts on native macroinvertebrate communities
}

\author{
Ivana Silva • Daniel Naya - Franco Teixeira de Mello - Alejandro D'Anatro • \\ Giancarlo Tesitore $\cdot$ Cristhian Clavijo $\cdot$ Iván González-Bergonzoni
}

Accepted: 23 March 2021 / Published online: 2 April 2021

(C) Springer Nature Switzerland AG 2021

\section{Correction to: Hydrobiologia}

https://doi.org/10.1007/s10750-020-04421-9

In the above mentioned publication, the surname of the third author appeared incorrectly as it should have read Teixeira de Mello. The original article has been corrected and the proper representation of the authors' names and their affiliation is also listed here.

Publisher's Note Springer Nature remains neutral with regard to jurisdictional claims in published maps and institutional affiliations.

A1 The original article can be found online at https://

A2 doi.org/10.1007/s10750-020-04421-9.

A3 I. Silva $(\varangle) \cdot$ I. González-Bergonzoni $(\square)$

A4 Departamento del Agua, CENUR Litoral Norte,

A5 Universidad de la República, ruta $3 \mathrm{~km} 363$ EEMAC,

A6 60000 Paysandú, Uruguay

A7 e-mail: yvanasilva92@gmail.com

\section{A8 I. González-Bergonzoni \\ A9 e-mail: ivg@fcien.edu.uy}

A10 I. Silva · D. Naya · A. D’Anatro .

A11 I. González-Bergonzoni

A12 Departamento de Ecología y Evolución, Facultad de

A13 Ciencias, Universidad de la República, Iguá 4225, Malvín

A14 Norte, 11400 Montevideo, Uruguay

A15 I. Silva · I. González-Bergonzoni

A16 Departamento de Ecología y Biología Evolutiva, Instituto

A17 de Investigaciones Biológicas, Clemente Estable, Av.

A18 Italia 3328, 11600 Montevideo, Uruguay
A19 F. Teixeira de Mello · G. Tesitore

A20 Departamento de Ecología y Gestión Ambiental CURE,

A21 Universidad de la República, Tacuarembó s/n,

A22 Maldonado, Uruguay

A23 C. Clavijo

A24 Museo Nacional de Historia Natural, 25 de Mayo 582,

A25 11000 Montevideo, Uruguay 